\title{
Velocity map imaging using an in-vacuum pixel detector
}

\author{
Georg Gademann, ${ }^{1, a)}$ Ymkje Huismans, ${ }_{1}^{1}$ Arjan Gijsbertsen, ${ }^{1}$ Julia Jungmann, ${ }^{1}$ \\ Jan Visschers, ${ }^{2}$ and Marc J. J. Vrakking ${ }^{1}$ \\ ${ }^{1}$ FOM-Institute for Atomic and Molecular Physics (AMOLF), Science Park 113, 1098 XG Amsterdam, \\ The Netherlands \\ ${ }^{2}$ National Institute for Sub-Atomic Physics (Nikhef), Science Park 105, 1098 XG Amsterdam, The Netherlands
}

(Received 7 July 2009; accepted 19 September 2009; published online 22 October 2009)

\begin{abstract}
The use of a new type in-vacuum pixel detector in velocity map imaging (VMI) is introduced. The Medipix 2 and Timepix semiconductor pixel detectors $\left(256 \times 256\right.$ square pixels, $\left.55 \times 55 \mu \mathrm{m}^{2}\right)$ are well suited for charged particle detection. They offer high resolution, low noise, and high quantum efficiency. The Medipix 2 chip allows double energy discrimination by offering a low and a high energy threshold. The Timepix detector allows to record the incidence time of a particle with a temporal resolution of $10 \mathrm{~ns}$ and a dynamic range of $160 \mu \mathrm{s}$. Results of the first time application of the Medipix2 detector to VMI are presented, investigating the quantum efficiency as well as the possibility to operate at increased background pressure in the vacuum chamber. () 2009 American Institute of Physics. [doi:10.1063/1.3246811]
\end{abstract}

\section{INTRODUCTION}

Scientific interest in the interaction of atoms and molecules with other atoms, ions, or electromagnetic radiation has led to the development of a wide range of imaging detectors. By detecting the spatial and temporal coordinates of charged particles and thus their velocities, detailed conclusions about the processes leading to the formation of the charged particles can be drawn. The most prevalent methods include velocity map imaging (VMI) spectrometers as well as cold target recoil ion momentum spectrometers, today in a broader understanding also referred to as reaction microscopes. Both techniques are based on similar working principles. Physical or chemical processes in a target are triggered either by particle impact or by the interaction with a light field, which results in charged fragments. The processes involved can include any form of direct or indirect ionization, dissociation, or even a chemical reaction. The charged fragments (ions and/or electrons) are accelerated in a dc electric field onto a two-dimensional (2D) detector where they are registered as events. Their positions and/or arrival times are recorded, giving insight into the angular and kinetic energy distribution.

VMI (see Fig. 1) is a high resolution ion or electron imaging technique. Here electrostatic optics, usually consisting of a flat repeller plate, a flat apertured extractor plate, and a flight tube with another flat and apertured ground plate are used. The geometry of these parts and the applied voltages determine the fragments' path of flight toward the detector. Their lateral displacement on the detector upon arrival is defined by the initial velocity vectors perpendicular to the detector axis. ${ }^{1-3}$ A unique feature of VMI lies in decoupling the position of creation of the fragments from their impact position on the detector by using an electrostatic lens. In this way, spatial blurring of the image is minimized and a veloc-

\footnotetext{
a)Electronic mail: g.gademann@amolf.nl.
}

ity resolution of less than $1 \%$ can be achieved. ${ }^{4}$ At the same time VMI maintains a linear mapping of the fragments' velocities across the detector. Under typical VMI focusing conditions, the spectrometer is operated under conditions which are close to the Wiley-McLaren time-of-flight focusing condition, decreasing the effects of the position of creation on the arrival time and thus creating a $2 \mathrm{D}$ projection of the original three-dimensional (3D) velocity distribution on the detector surface. By choosing the axis of symmetry in the interaction region parallel to the plane of the detector, the original 3D distribution can be retrieved with appropriate numerical algorithms. In VMI measurements, several thousand events per laser shot can usually be recorded before detrimental effects such as changes to the detected velocities as a result of space charge set in, and an image composed of many individual hits forms the final measurement.

The VMI experiments typically only yield velocity components of a single either negatively or positively charged fragment at a time. Thus they offer only an incomplete view of the reactants' full momentum distribution. In order to retrieve the full information from an experiment, the momentum vectors of all participating fragments-negatively as well as positively charged-have to be measured in coincidence. Such kinematically complete experiments can be accomplished by using reaction microscopes. ${ }^{5,6}$ Positively charged fragments are mapped by a weak homogeneous electric field onto a position- and time-sensitive detector. The time and place of incidence then allows to reconstruct the initial momentum of these fragments. Electrons are accelerated by the same electric field in the opposite direction. The electron velocities are much higher than those of the ions due to their smaller mass. To account for this difference, an additional weak magnetic field is often applied which guides them on a spiral-shaped track onto a second detector. The retrieval of the initial momentum components of all fragments over a solid angle of almost $4 \pi$ is possible. The signal rates in reaction microscope measurements have to be small 


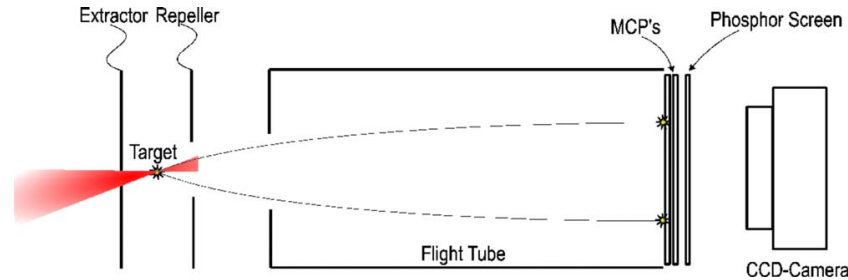

FIG. 1. (Color online) Schematic of a VMI setup showing the repeller and extractor electrodes for applying the acceleration voltages which project the charged particles onto the MCP detector.

enough to allow unambiguous coincidence recognition. Hence, the use of a detector with high quantum efficiency is crucial.

In both cases, VMI and reaction microscopes, the conventional charged particle detectors consist of multiple microchannel plates (MCPs) for signal amplification. They are followed by either phosphor screens in combination with charge-coupled device (CCD) cameras, delay line anodes or wedge-and-strip anodes. ${ }^{7}$ Due to the active open surface of MCPs, the quantum efficiencies of such setups are limited to around $60 \%$.

In the case of phosphor/CCD setups the electron cloud leaving the MCPs hits the phosphor screen and locally creates photons, which then are optically registered by the CCD camera where they are transformed into an electronic signal. It follows that this is a rather indirect detection process. These photons again create electron-hole pairs which finally lead to the detected signal. CCD cameras produce good spatial resolution, especially when using centroiding algorithms,${ }^{8}$ and are available for reasonable prices. A drawback is that there is no submicrosecond timing information available, which can only be obtained by using expensive and complicated pulsed gates.

When using wedge-and-strip or delay line anodes, the position can be reconstructed by measuring the relative charge distribution in the former and relative timing signals in the latter case. Commercially available detector setups allow spatial resolutions down to $50 \mu \mathrm{m},{ }^{9}$ while timing information extracted directly from the MCPs can reach $<300$ ps. $^{10}$ The dead time of delay line anodes of $\sim 20 \mathrm{~ns}$ is short compared to that of charge integrating anodes, which cannot spatially resolve events with a time difference below several hundred nanoseconds. ${ }^{11}$ Both reconstructions nevertheless require involved electronic processing as well as a slow event-by-event transfer to the computer, which limits the event acquisition rate to maximally $200 \mathrm{kHz} .^{10}$ The above-mentioned numbers apply to the most commonly used setups in experimental laboratories. Better results have been obtained, at the cost of extremely dedicated and complex setups. For further reference see Refs. 12 and 13.

A problem that we encountered in our own research are restrictions imposed by the maximum tolerable operating pressure of an MCP. MCPs cannot be operated at pressures higher than $10^{-5}$ mbar. In many experiments such a maximum pressure is perfectly tolerable, however, exceeding this value may become desirable in experiments where the collision rate is very low due to cross sections and/or the flux of incoming particles (photons and ions) being very small. Here

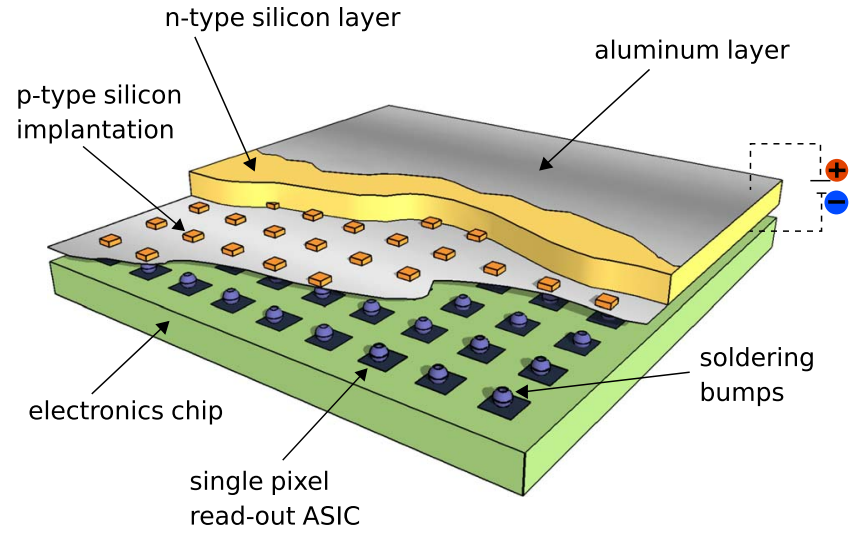

FIG. 2. (Color online) Schematic overview of the Medipix 2 detector. Visible are the sensor layer for electron-hole pair creation and the applied bias voltage as well as the bump-bonded readout ASIC.

one would like to increase the target gas densities, which, at a constant pumping speed, can increase the background pressure above the tolerable limit. For this reason, we have started investigating the possibility to use alternative detector types in VMI and/or reaction microscopy setups.

Here we propose a new concept, moving to an invacuum pixel detector which has the potential to circumvent most of the above-mentioned limitations. After an introduction to this new detector type, a range of possible applications will be described. Subsequently first experimental results achieved using this detector will be presented.

\section{THE MEDIPIX/TIMEPIX CHIPS}

The Medipix 2 detector is a pixel detector that was designed by the CERN Electronics Department on behalf of the MEDIPIX collaboration. ${ }^{14}$ It was originally designed for counting X-ray photons, without noise or dark current, at high fluxes (several gigaphotons per $\mathrm{cm}^{2}$ per second). At the same time good position resolution is achieved using 256 $\times 256$ pixels with a pitch of $55 \mu \mathrm{m}$. The Medipix 2 detector is the successor of the earlier Medipix 1 chip, consisting of $64 \times 64$ pixels with a pixel size of $170 \times 170 \mu \mathrm{m}^{2}$. In its normal operation mode, the Medipix 2 detector uses a pixelated array of reverse-biased semiconducting diodes as a sensor layer which is bump bonded to the Medipix2 readout application specific integrated circuit (ASIC), ${ }^{15,16}$ as seen in Fig. 2.

In our case a $300 \mu \mathrm{m}$ thick array of reverse-biased photodiodes is used as the sensor. It is made from slightly $n$-doped high-resistivity silicon, with a $p$-type implantation in every pixel. The entrance window on the $n$ side is an Ohmic contact of $150 \mathrm{~nm}$ thick aluminum, where the bias voltage is applied. In the sensor layer every impinging particle creates one electron-hole pair per $3.6 \mathrm{eV}$ of deposited energy. When a positive voltage is applied to the unsegmented $n+$ contact the holes generated drift toward the pixelated $p+$ contacts for collection. The amount of charge that the pixel electronics will detect is therefore proportional to the energy deposited in the detector layer by the impinging particle. With the sensor layer described above photons and electrons can efficiently be detected, provided that the pho- 


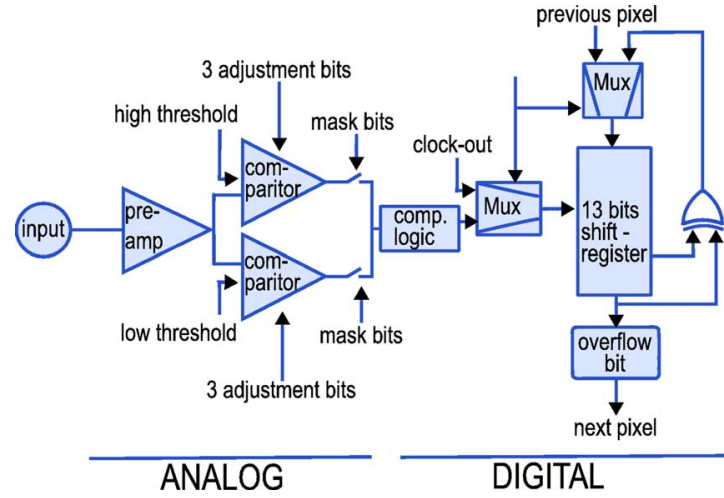

FIG. 3. (Color online) Schematic of the readout electronics. Every pixel consists of an analog amplifier part and the digital part containing threshold discrimination, individual adjustment bits, as well as the counter. Using the adjustment bits, a uniform detector response over the complete chip is ensured. This equalization can be automatically performed using the available readout software.

ton, respectively, electron kinetic energy exceeds the $4 \mathrm{keV}$ detection threshold. For electrons detected in a VMI, this requirement can easily be met. With the sensor layer described above ions cannot be detected since ions will not penetrate the aluminum entrance layer. Therefore for ion detection one has to rely on different means of charge amplification, as described in a later paragraph of this paper. We note that so far detection efficiencies of $85 \%$ have been shown experimentally. ${ }^{17}$

The ASIC contains in each individual pixel an analog input stage, an electronic shutter, two individually tunable energy discriminators, as well as a 14 bit pseudorandom counter (see Fig. 3). By making use of the two discriminator levels one can define an energy window, such that only incident particles within this window will lead to a registered event. Since the threshold energies that can be chosen for the discriminator levels lie well above the noise level of the pixels $\left(\sim 100 e^{-}\right)$, almost noise free measurements are possible. ${ }^{18}$ There are three further adjustment bits available for every pixel, which are used to equalize the detector response over the full pixel array. This feature is easily accessible via a software routine from the openly available control program PIXELMAN (Refs. 19 and 20), which is run on a PC. Connection to the chip is established either via a USB interface ${ }^{21,22}$ or a serial interface. ${ }^{23,24}$

The parameters, such as acquisition time and number of acquisitions, can easily be controlled by the same software. Here the acquisition window can be selected ranging from tenths of a second to several minutes or hours. The maximum single pixel acquisition rate for consecutive events lies at approximately $100 \mathrm{kHz}$ per pixel. This means that during the chosen acquisition window events arriving on a single pixel with temporal distance larger than $10 \mu$ s will be separately counted. For separate pixels parallel detection over the complete chip surface is possible. Therefore a maximum of 65536 separate events can be registered at the same time, assuming they are detected on separate pixels. This is an obvious advantage over wedge-and-strip or delay line anodes, where at most one or a few incoming particles can be tolerated on the entire detector in one readout cycle. ${ }^{11}$
The Timepix chip was conceived as a small modification of the Medipix2 readout chip ${ }^{23,25}$ and is based on the same technological principles. In addition to the normal eventcounting mode of the Medipix 2 chip, it is especially adopted to measure not only the position but also the arrival time of the incoming particle. Each Timepix pixel can be programmed in one of three modes: single particle counting (like Medipix2), arrival time (counting clock ticks from the moment the particle arrives until the shutter closes), or time over threshold (counting clock ticks as long as the discriminator threshold is exceeded). The Timepix pixels have only one energy discrimination threshold, thereby still allowing noise free detection. The time over threshold output is directly proportional to the amount of charge deposited on the particular pixel, i.e., to the energy of the detected particle. This is an option that is very important when centroiding algorithms are applied to achieve subpixel spatial resolution for events that lead to a detectable signal over a range of pixels, such as the signals that can be detected when electron or ion signals are first amplified using a mcirochannel plate detector placed in front of the Timepix detector, ${ }^{26,27}$ as will be further explained in a later paragraph.

The general idea of Timepix is to distribute an external clock to the full $256 \times 256$ pixel matrix. The pixel logic is changed such that the counting of clock pulses in the pseudorandom register is enabled by the first hit after the shutter opens and continues until the shutter closes. The nominal clock frequency for Medipix2 using the so-called MUROS interface $^{24}$ is $160 \mathrm{MHz}$. This clock signal is an external input to the chip and can be varied at will. In this way every pixel gets a "common-stop time to digital converter (TDC)" with $10 \mathrm{~ns}$ resolution and $2^{14} \times 10 \mathrm{~ns}=160 \mu \mathrm{s}$ dynamic range. Only a small number of pixels will typically be "hit" during an exposure so most of the counters will have a zero-value during readout. After the acquisition is completed, all the $\sim 65000$ pixel counter contents are serially shifted out in 5-10 ms. By using the 32 bit parallel readout bus of the chip one can obtain frame rates of $\sim 1 \mathrm{kHz} .^{28}$

Both Timepix and Medipix 2 chips $^{29}$ are buttable from three sides without creating any dead spaces in between the single chips. One edge is reserved for electronic readout via wire bonds. In this way detector arrays of $2 \times n$ chips can be created. For the presented experiments an array of $2 \times 2$ chips was used, creating an active detection area of 28 $\times 28 \mathrm{~mm}^{2}$, which will be referred to as the Medipix2 Quad detector. In the framework of the RELAXD project the development of electronic readout using vias through the chip and 1 gigabit/s bandwidth will facilitate the production of even larger detector arrays. ${ }^{30}$ As a followup to the Medipix2 detector, the Medipix3 chip has already been designed at CERN and is currently being tested. ${ }^{31}$ A successor for the Timepix chip is still in the design phase.

In applications like VMI the Medipix2 or Timepix chip in combination with a suitable sensor layer can easily be implemented. Without further optimization it can be used to image electrons with high efficiency and good spatial resolution. The data is immediately available in ASCII or binary formats. Whereas MCP detectors offer efficiencies of around $60 \%$, corresponding to the active surface, Medipix 2 detectors 
have been shown to reach efficiencies up to at least $85 \%$ and are expected to achieve an even unit detection efficiency. At the same time Medipix2 chips offer an even efficiency distribution over the whole active surface.

\section{ENVISIONED RANGE OF APPLICATION}

\section{A. VMI}

As mentioned above, the first considerations to use Medipix 2 chips in our research were initiated by the pressure limitations imposed by MCP-based detectors. So far there are no such limitations known for Medipix 2 chips other than the effects of electrical breakdown as described by the Paschen curve. In 2D VMI, high signal rates are desirable since typically one would like to record a projected image with $10^{5}-10^{6}$ events in order to be able to perform the extraction of the 3D velocity distribution with high accuracy. Depending on the available light source or projectile that is used in the collision experiment, achieving this number in a reasonable time can be challenging. In our own attosecond experiments, where photon numbers can be as low as $10^{5}$ per shot and experimental conditions rigorously have to be kept stable, this can be hard to do. Therefore efficient use of the available photons has to be made, for example, by increasing the target gas density. However, this also increases the background pressure in the apparatus. Whereas this approach fails for MCPs, Medipix 2 can be operated over a large pressure range reaching from high vacuum to atmosphere.

The Medipix 2 chip is thus expected to allow an increase in signal rate by as much as one to two orders of magnitude, thus decreasing the acquisition time by the same factor. In the remainder of this paper we will present a successful demonstration of this concept. First, however, we describe a few other applications of the Medipix2/Timepix chip in VMI and/or reaction microscopy which we regard as very promising and soon hope to develop.

\section{B. 3D measurements}

Time as the third dimension in imaging experiments can be accessed by using the Timepix instead of the Medipix 2 chip. This will permit ion time-of-flight measurements simultaneously to VMI measurements. Nowadays in VMI, such measurements are already performed by applying slice imaging. ${ }^{4,32}$ Gating the detector and recording a series of $2 \mathrm{D}$ velocity distributions, the original 3D distribution of the particles can be retrieved. This, however, requires sophisticated timing and gating electronics to operate the detector. The application of the Timepix chip allows direct measurements with an arrival time resolution down to $10 \mathrm{~ns}$. First tests of this capability by measuring the difference in arrival time of backward and forward emitted ion fragments with respect to the detector are in progress in our laboratory.

\section{High position resolution measurements}

High resolution photon counting using the Timepix chip has been performed and described by Vallerga et al. ${ }^{26}$ The sensor diode matrix above the readout ASIC was replaced by an MCP, which also allows the detection of positively charged ions. The resulting charge cloud behind the MCP is detected on the pixel input pad of the bare Medipix2/Timepix readout ASIC. Depending on the distance and extraction voltage between the MCP and the Timepix ASIC, the charge cloud coming from the MCP will cover multiple pixels. Using the above-mentioned ability to determine the amount of charge deposited per pixel, a weighted centroiding algorithm can be used to achieve spatial resolution well below $10 \mu \mathrm{m} .{ }^{27}$ In this case the size of the MCP pores becomes the limiting factor. A similar technique has been known and applied also in VMI measurements for quite some time. Centroiding pixel counts on CCD cameras also leads to an improvement of spatial resolution with current MCP-phosphor combinations. ${ }^{8}$

\section{Coincidence measurements}

In combination with reaction microscopes, the Timepix detectors promise experiments with considerably less technological effort. Higher spatial accuracy as well as faster readout can be achieved by combining techniques described above. Precise timing information still has to be obtained from the fast MCP signal, whereas the Timepix temporal information can deliver a time stamp for the recorded event to unambiguously recognize coincidences on multiple detectors. In this way coincidence measurements at increased count rates are feasible.

\section{FIRST TESTS USING THE MEDIPIX2 CHIP}

In this section we report the first application of a Medipix 2 chip assembly to VMI. The following measurements show that the detector is capable of replacing the commonly used 2D MCP-based charged particle detectors in such experiments. The most important aspects of the new detector such as spatial resolution, quantum efficiency, and behavior for different background pressures have been tested. As a first experiment we recorded multiphoton ionization signals from xenon (see Fig. 4). The third harmonic at $355 \mathrm{~nm}$ (3.49 $\mathrm{eV}$ ) of a flash lamp pumped yttrium aluminum garnet (YAG) laser was used as the pulsed laser source. The intensity of the laser pulses is estimated to reach $10^{12} \mathrm{~W} / \mathrm{cm}^{2}$ at a repetition rate of $10 \mathrm{~Hz}$. The acquisition window of the Medipix 2 chip was chosen to be $0.1 \mathrm{~s}$ to ensure that for each acquisition only the signal of one laser pulse was recorded. Depending on the signal strength 100-1000 acquisitions were summed up to produce the image. We were able to observe the signal from two different four-photon ionization processes, corresponding to the ${ }^{2} P_{3 / 2}(12.13 \mathrm{eV})$ and ${ }^{2} P_{1 / 2}(13.44 \mathrm{eV})$ ionization thresholds of Xe. ${ }^{33}$ The kinetic energies of the detected electrons were therefore 1.83 and $0.52 \mathrm{eV}$. These kinetic energies were measured with an energy resolution $\Delta E / E$ better than 0.07 and 0.03 , respectively (see Fig. 6).

We will discuss the individual measurements and results in detail in the following paragraphs. The most important aspect of this work was to test the capability of Medipix 2 to increase the signal rate of VMI experiments. Therefore the quantum efficiency and the ability to operate the detector at elevated background pressures were investigated. 


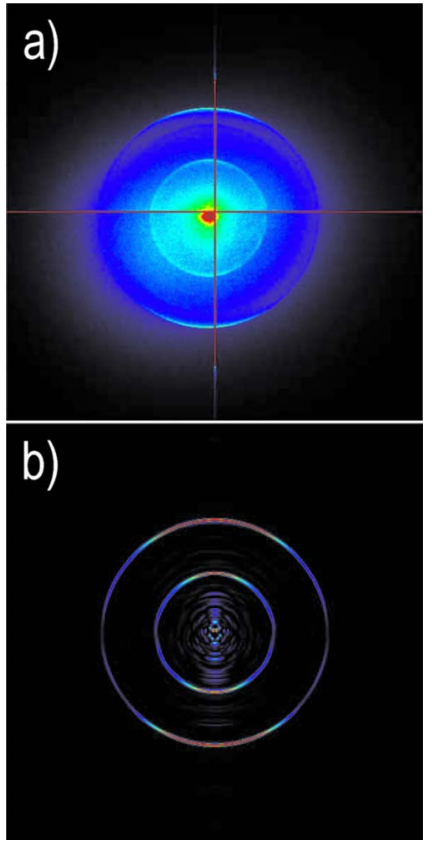

FIG. 4. (Color online) (a) Photoelectron image for four-photon ionization of Xe by a $355 \mathrm{~nm}$ Nd:YAG laser, recorded by the Medipix2 chip. Considerable signal due to ionization of background gas is also visible. The innerand outermost rings are due to the xenon signal which selectively was obtained by background subtraction. Since the images were recorded using a Medipix2 Quad detector, a bright cross is visible which is caused by the larger surface pixels at the joining sides of the four readout ASICs that read the quad sensor. These pixels can either be masked or adjusted for the increased size after the measurement. (b) After background subtraction an inversion algorithm allows to extract a slice through the original 3D distribution, thereby revealing the velocity and angular distribution of the ejected photoelectrons.

\section{A. Quantum efficiency}

The efficiency of the Medipix2 detector will mainly depend on the kinetic energy of the particles to be detected and on the energy thresholds chosen in the discriminator logic. We expect to see a rise in detected signal, as a function of the acceleration voltage of the electrons. This acceleration voltage can be chosen by applying a higher voltage to the repeller and extractor plates of the VMI setup while maintaining electron focusing conditions at the detector surface. As described above, the detection threshold lies at approximately 4 $\mathrm{keV}$. This level may vary depending on the amount of leakage current in the detector which itself depends on temperature as well as on the amount of scattered light hitting the detector surface. In the present experiments, where a large flux of ultraviolet $355 \mathrm{~nm}$ photons was used to create an electron signal, the latter was definitely an issue and grounds for further improvements in the future.

Two measurements were conducted, varying the voltage of the VMI setup from $4 \mathrm{kV}$ to almost $17 \mathrm{kV}$, as shown in Fig. 5. There is a shift in the two curves in voltage dependence. This shift is due to different threshold levels used in the two measurements, i.e., the threshold necessary to suppress noise stemming from scattered UV light hitting the detector (see above). In the second experiment, more light hit the detector and therefore increased the dark counts. Consequently, a higher threshold level had to be chosen.

The curves nevertheless show the same qualitative fea-

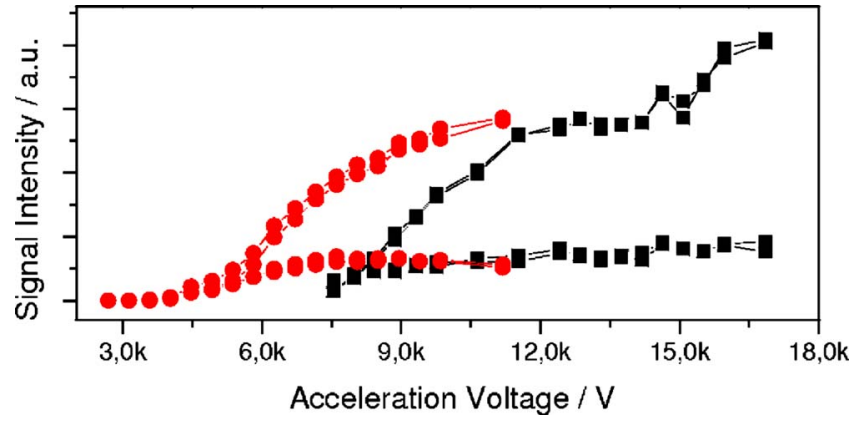

FIG. 5. (Color online) Voltage dependence of the signal rate for two different setups (red and black curves). The shift between the two curves is due to different threshold settings in both cases and can be explained by the amount of scattered light reaching the detector in the two measurements. However the appearance of the same qualitative features should be noted. At voltages around $10 \mathrm{kV}$ a plateau region becomes apparent. The subsequent rise is due to charge-sharing effects.

tures. At acceleration voltages of around $10 \mathrm{kV}$, a plateau region becomes apparent, which we interpret as a sign that unit detection efficiency has been achieved. In the second experiment the voltage range was increased to investigate the extent of this plateau region. Eventually a second increase in signal was observed at even higher acceleration voltages. At such high impact energies impinging particles create very large charge clouds that laterally spread on their path through the sensor layer, leading to detection on multiple adjacent pixels. This effect is known as charge-sharing leading to higher signal counts. ${ }^{34,35}$ Charge-sharing can readily be recognized in single-shot acquisitions where multiple adjacent pixels show counts simultaneously. Depending on the thickness of the sensor layer and the applied bias voltage this behavior can be influenced. As discussed above in the case of using MCPs in combination with bare Medipix2/Timepix chips, this effect can even be desirable and exploited for centroiding.

In conclusion, measurements were performed suggesting that unit quantum efficiency can be achieved and the necessary range of acceleration voltages was identified. This range is expected to extend to lower acceleration voltages as we move to other laser sources, such as femtosecond and/or extreme ultraviolet (XUV) light sources. Here the required light intensities can be reached at much lower single pulse energies thus decreasing the amount of scattered light hitting the detector surface. Accordingly adjusting the detection threshold levels will lead to unit quantum efficiency at considerably lower impact energies.

\section{B. Operation at increased background pressure}

A second test was performed to investigate the pressure operating range for Medipix2. As mentioned above, the expectation is to be able to perform VMI measurements with a 10-100 times increased target gas density. The base pressure in the experimental setup was in the range of $10^{-7} \mathrm{mbar}$, in the presence of the target gas jet. To increase the background pressure to the required testing levels, a leak valve was installed through which the chamber pressure could be precisely controlled. Helium was chosen as leak gas to increase the background pressure without affecting the signal level. In 

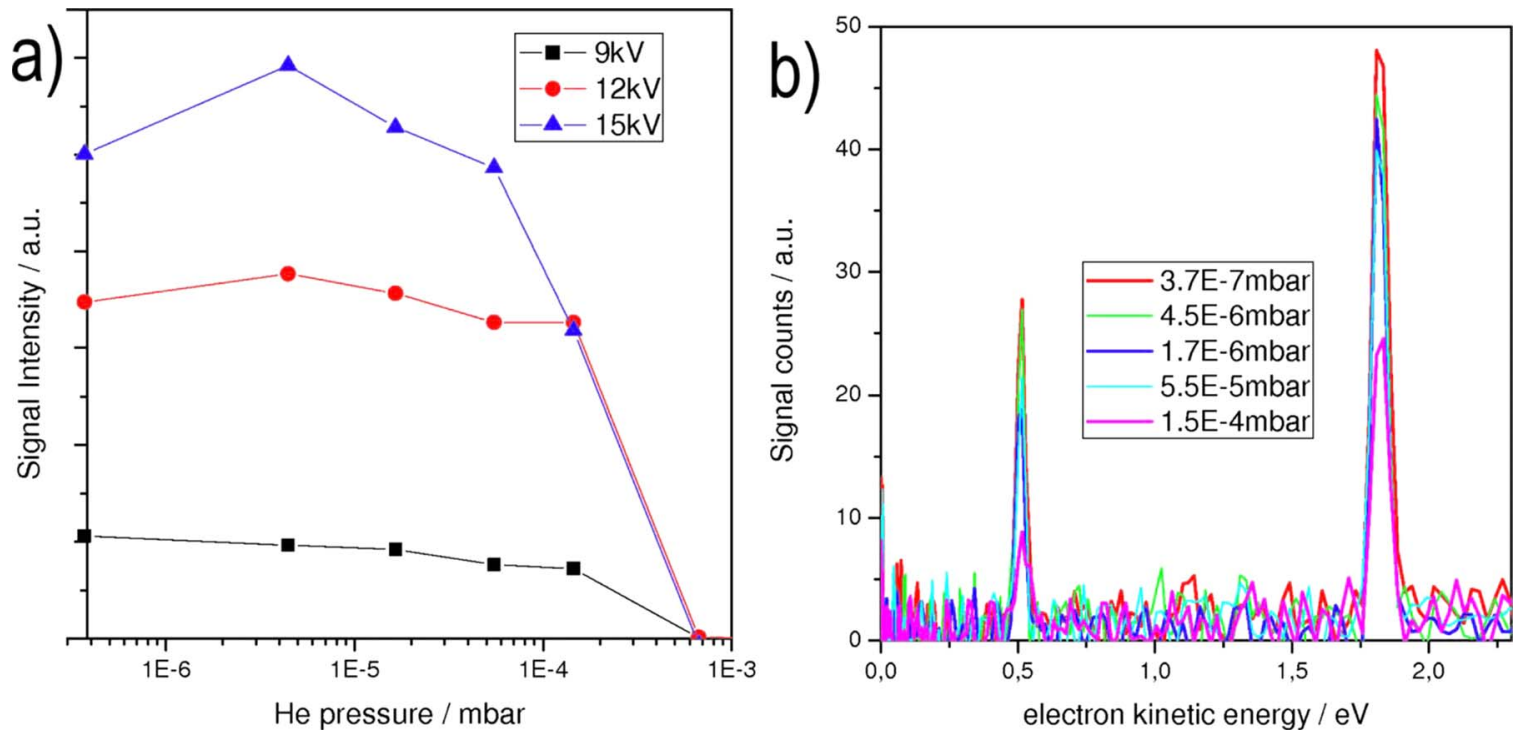

FIG. 6. (Color online) (a) The pressure dependence of the signal intensity is shown for different acceleration voltages. A clear decrease in signal with increasing background pressure is visible. For pressures above $10^{-3}$ mbar the mean free pathlength for electrons in the extraction region of the VMI (see Fig. 1) becomes too small for a reliable measurement. (b) Even though we see a reduction in signal due to the decreasing mean free path length, the kinetic energy resolution of the detector is maintained over the full pressure range.

this way it was possible to separate the influence of high pressure on the electron generation process and the subsequent detection. The pressure was increased in steps to $\sim 10^{-3}$ mbar. Above this pressure the operation becomes restricted by the turbo molecular pumps. Another restriction that has to be respected is the electric breakdown of gases as described by the Paschen curve. In order to protect the chip assembly and prevent electronic breakdown over the sensor layer, gas pressures exceeding $10^{-3}$ mbar were avoided.

The Xe target gas densities were held at a constant level throughout the tests. Therefore any drop in signal stems solely from the increased background pressure. Of course, in a real application of the detector at elevated pressure the target gas density would be increased to the maximum tolerable amount.

In Fig. 6 a moderate decrease in signal for pressures above $10^{-5}$ mbar followed by a much more rapid drop above a background pressure of $10^{-4} \mathrm{mbar}$ can be seen. Above pressures of $10^{-3}$ mbar the signal becomes too weak for decent measurements. At the same time the original spatial resolution is maintained throughout the complete tested pressure range. This behavior is attributed to the reduced mean free pathlength especially for slow electrons during acceleration. As electrons are liberated in the laser focus, their initial velocity is on the order of a couple of $\mathrm{eV}$. In the tested pressure range the mean free path for such slow electrons lies between 0.5 and $500 \mathrm{~mm}$. This is about four orders of magnitude lower than the mean free path for electrons traveling with a kinetic energy of $5 \mathrm{keV}^{36}$ Collisions of slow electrons with the background helium atoms will lead to a strong deviation from their original path, preventing them from reaching the detector. After acceleration, the electrons will travel with less disturbance. Therefore no spreading or blurring of the signal is visible.

\section{CONCLUSION}

In conclusion, we have reported in this paper a first example of the use of pixel detectors for position-sensitive ion/ electron detection. Given the prevalence of these types of detectors in chemistry and physics research and given the large as yet unexplored potential of these detectors, we foresee widespread use of these detectors in the coming years, where the results presented here merely serve as a first illustration.

\section{ACKNOWLEDGMENTS}

The authors would like to thank the Medipix Consortium for providing the Medipix2 detector assembly as well as acknowledge helpful discussions with Jan Visser, Martin Fransen, Daan Boltje, Lukas Tomasek, and technical support from Joop Rovekamp. This work is part of the research program of the "Stichting voor Fundamenteel Onderzoek der Materie (FOM)," which is financially supported by the "Nederlandse organisatie voor Wetenschappelijk Onderzoek (NWO)."

${ }^{1}$ D. W. Chandler and P. L. Houston, J. Chem. Phys. 87, 1445 (1987)

${ }^{2}$ A. J. R. Heck and D. W. Chandler, Annu. Rev. Phys. Chem. 46, 335 (1995).

${ }^{3}$ A. Eppink and D. Parker, Rev. Sci. Instrum. 68, 3477 (1997).

${ }^{4}$ V. Papadakis and T. Kitsopoulos, Rev. Sci. Instrum. 77, 083101 (2006).

${ }^{5}$ R. Moshammer, M. Unverzagt, W. Schmitt, J. Ullrich, and H. SchmidtBöcking, Nucl. Instrum. Methods Phys. Res. B 108, 425 (1996).

${ }^{6}$ J. Ullrich, R. Moshammer, A. Dorn, L. Schmidt, and H. Schmidt-Böcking, Rep. Prog. Phys. 66, 1463 (2003).

${ }^{7}$ D. de Bruijn and J. Los, Rev. Sci. Instrum. 53, 1020 (1982).

${ }^{8}$ B.-Y. Chang, R. Hoetzlein, J. Mueller, J. Geiser, and P. Houston, Rev. Sci. Instrum. 69, 1665 (1998).

${ }^{9}$ R. Dörner, V. Mergel, O. Jagutzki, L. Spielberger, J. Ullrich, R. Moshammer, and H. Schmidt-Böcking, Phys. Rep. 330, 95 (2000).

${ }^{10}$ RoentDek Handels GmbH, Kelkheim-Ruppertshain, Germany.

${ }^{11}$ V. Mergel, O. Jagutzki, L. Spielberger, K. Ullmann-Pfleger, R. Dörner, and H. Schmidt-Böcking, Mater. Res. Soc. Symp. Proc. 502, 171 (1998). 
${ }^{12}$ J. Vallerga, J. McPhate, A. Tremsin, and O. Siegmund, Astrophys. Space Sci. 320, 247 (2009)

${ }^{13}$ J. Lapington, Nucl. Instrum. Methods Phys. Res. A 525, 361 (2004).

${ }^{14}$ Homepage of the Medipix Collaboration www.cern.ch/medipix.

${ }^{15}$ X. Llopart, M. Campbell, R. Dinapoli, D. S. Segundo, and E. Pernigotti, IEEE Trans. Nucl. Sci. 49, 2279 (2002).

${ }^{16}$ X. Llopart and M. Campbell, Nucl. Instrum. Methods Phys. Res. A 509, 157 (2003).

${ }^{17}$ G. MacMullan, D. Cattermole, S. Chen, R. Henderson, X. Llopart, C. Summerfield, L. Tlustos, and A. Faruqi, Ultramicroscopy 107, 401 (2007).

${ }^{18}$ A. Faruqi, R. Henderson, and L. Tlustos, Nucl. Instrum. Methods Phys. Res. A 546, 160 (2005).

${ }^{19}$ T. Holy, J. Jakubek, S. Pospisil, J. Uher, D. Vavrik, and Z. Vykydal, Nucl. Instrum. Methods Phys. Res. A 563, 254 (2006).

${ }^{20}$ Czech Technical University of Prague, IEAP, Pixelman software homepage aladdin.utef.cvut.cz/ofat/others/Pixelman/index.

${ }^{21}$ Z. Vykydal, J. Jakubek, and S. Pospisil, Nucl. Instrum. Methods Phys. Res. A 563, 112 (2006).

${ }^{22}$ Czech Technical University in Prague, IEAP, MedipixTimepix USB-interface homepage aladdin.utef.cvut.cz/ofat/others/ USB_interface/index.html.

${ }^{23}$ D. SanSegundo Bello, M. van Beuzekom, P. Jansweijer, H. Verkooijen, and J. Visschers, Nucl. Instrum. Methods Phys. Res. A 509, 164 (2003).

${ }^{24}$ National Institute for Sub-Atomic Physics (Nikhef), MUROS-interface project homepage www.nikhef.nl/pub/experiments/medipix/muros.html.

${ }^{25}$ H. van der Graaf, J. Timmermans, J. Visschers, M. Campbell, Paul Colas, Timepix Proposal Nov. 2004 www.nikhef.nl/pub/experiments/medipix/ TimePix.pdf.

${ }^{26}$ J. Vallerga, J. McPhate, A. Tremsin, O. Siegmund, B. Mikulec, and A.
Clark, Nucl. Instrum. Methods Phys. Res. A 546, 263 (2005).

${ }^{27}$ B. Mikulec, A. Clark, D. Ferrére, D. L. Marra, J. McPhate, O. Siegmund, A. Tremsin, J. Vallerga, J. Clément, C. Ponchut, and J.-M. Rigal, Nucl. Instrum. Methods Physics Res. A 567, 110 (2006).

${ }^{28}$ X. Llopart, R. Ballabriga, M. Campbell, L. Tlustos, and W. Wong, Nucl. Instrum. Methods Phys. Res. A 581, 485 (2007).

${ }^{29}$ Research licenses to obtain the Medipix 2 and/or Timepix chip, with a corresponding USB readout system, and PIXELMAN software package, can be obtained via the chairperson of the Medipix2 Collaboration, Michael Campbell from PH/ESE, CERN, Geneva, Switzerland. Tel: +41 2276 74866, Email: michael.campbell@cern.ch.

${ }^{30}$ Z. Vykydal, J. Visschers, D. Sabuncuoglu Tezcan, K. de Munck, T. Borgers, W. Ruythooren, and P. de Moor, Nucl. Instrum. Methods Phys. Res. A 591, 241 (2008).

${ }^{31}$ Medipix Collaboration, Medipix 3 project homepage http://medipix.web.cern.ch/MEDIPIX/Medipix3/homeMP3.htm.

${ }^{32}$ M. Lipciuc, J. Buijs, and M. H. M. Janssen, Phys. Chem. Chem. Phys. 8, 219 (2006).

${ }^{33}$ P. Kruit, J. Kimman, H. Muller, and M. van der Wiel, Phys. Rev. A 28, 248 (1983).

${ }^{34}$ M. Chmeissani, M. Maiorino, G. Blanchot, G. Pellegrini, J. Garcia, M. Lozano, R. Martinez, C. Puigdengoles, and M. Ullan, Proceedings of the IMTC 2004-Instrumentation and Measurement Technology Conference, 2004 (unpublished).

${ }^{35}$ M. Campbell, E. Heijne, T. Holy, J. Idàrraga, J. Jakubek, C. Lebel, C. Leroy, X. Llopart, S. Pospisil, L. Tlustos, and Z. Vykydal, Nucl. Instrum. Methods Phys. Res. A 591, 38 (2008).

${ }^{36}$ A. Jablonski, F. Salvat, and C. Powell, Standard Reference Data Program Database 64 (National Institute of Standards and Technology). 